\title{
Distinguishing Number of Countable Homogeneous Relational Structures
}

\author{
C. Laflamme * \\ University of Calgary \\ Department of Mathematics and Statistics \\ 2500 University Dr. NW. Calgary Alberta Canada T2N1N4 \\ laf@math.ucalgary.ca \\ L. Nguyen Van Thé ${ }^{\dagger}$ \\ Université Paul Cézanne - Aix-Marseille III \\ Avenue de l'escadrille Normandie-Niémen \\ 13397 Marseille Cedex 20, France \\ lionel@latp.univ-mrs.fr \\ N. Sauer \\ Department of Mathematics and Statistics \\ The University of Calgary, Calgary \\ Alberta, Canada T2N1N4 \\ nsauer@math . ucalgary.ca
}

Submitted: Apr 18, 2008; Accepted: Jan 20, 2010; Published: Jan 29, 2010

Mathematics Subject Classification: 05E18, 05C55, 05C15

\begin{abstract}
The distinguishing number of a graph $G$ is the smallest positive integer $r$ such that $G$ has a labeling of its vertices with $r$ labels for which there is no non-trivial automorphism of $G$ preserving these labels.

In early work, Michael Albertson and Karen Collins computed the distinguishing number for various finite graphs, and more recently Wilfried Imrich, Sandi Klavžar and Vladimir Trofimov computed the distinguishing number of some infinite graphs, showing in particular that the Random Graph has distinguishing number 2.

We compute the distinguishing number of various other finite and countable homogeneous structures, including undirected and directed graphs, and posets. We show that this number is in most cases two or infinite, and besides a few exceptions conjecture that this is so for all primitive homogeneous countable structures.
\end{abstract}

\footnotetext{
*Supported by NSERC of Canada Grant \# 690404

†The author would like to thank the support of the Department of Mathematics \& Statistics Postdoctoral Program at the University of Calgary

${ }^{\ddagger}$ Supported by NSERC of Canada Grant \# 691325
} 


\section{Introduction}

The distinguishing number of a graph $G$ was introduced in [1] by Michael Albertson and Karen Collins. It is the smallest positive integer $r$ such that $G$ has a labeling of its vertices into $r$ labels for which there are no non-trivial automorphism of $G$ preserving these labels. The notion is a generalization of an older problem by Frank Rubin, asking (under different terminology) for the distinguishing number of the (undirected) $n$-cycle $C_{n}$. It is interesting to observe that the distinguishing number of $C_{n}$ is 3 for $n=3,4,5$, and 2 for all other integer values of $n>1$. From these early days much research has been done on the distinguishing number of finite graphs. Of more interest to us here is the recent work of Wilfried Imrich, Sandi Klavžar and Vladimir Trofimov in [9] where they computed the distinguishing number of some infinite graphs, showing in particular that the Random Graph has distinguishing number 2.

In this paper we further generalize the notion to relational structures and compute the distinguishing number of many finite and countable homogeneous structures, including undirected and directed graphs, making use of the classifications obtained by various authors. We find that the distinguishing number is "generally" either 2 or $\omega$, and conjecture that this is the case for all countable homogeneous relational structures whose automorphism groups are primitive.

In the remainder of this section we review the standard but necessary notation and background results.

Let $\mathbb{N}=\omega \backslash\{0\}$ be the set of positive integers and $n \in \mathbb{N}$. An $n$-ary relation on a set $A$ is a set of $n$-tuples $R \subseteq A^{n}$. A signature is a function $\mu: I \rightarrow \mathbb{N}$ from an index set $I$ into $\mathbb{N}$, which we often write as an indexed sequence $\mu=\left(\mu_{i}: i \in I\right)$. A relational structure with signature $\mu$ is a pair $\mathfrak{A}:=\left(A, \mathbf{R}^{\mathfrak{A}}\right)$ where $\mathbf{R}^{\mathfrak{A}}:=\left(R_{i}^{\mathfrak{A}}\right)_{i \in I}$ is a set of relations on the domain $A$, each relation $R_{i}^{\mathfrak{A}}$ having arity $\mu_{i}$. An embedding from a structure $\mathfrak{A}:=\left(A, \mathbf{R}^{\mathfrak{A}}\right)$ into another structure $\mathfrak{B}:=\left(B, \mathbf{R}^{\mathfrak{B}}\right)$ of the same signature $\mu$ is a one-one map $f: A \rightarrow B$ such that for each $i \in I$ and $a \in A^{\mu_{i}}, a \in R_{i}^{\mathfrak{A}}$ iff $f(a) \in R_{i}^{\mathfrak{B}}$. An isomorphism is a surjective embedding, and an automorphism is an isomorphism from a structure to itself.

If $\mathfrak{A}$ is clear from the context then we will write $\mathbf{R}$ instead of $\mathbf{R}^{\mathfrak{A}}$ and $R_{i}$ instead of $R_{i}^{\mathfrak{A}}$. We also write $\mathfrak{A}:=(A, R)$ if there is only one relation $R$.

Let $\mathfrak{A}=(A, \mathbf{R})$ be a relational structure with automorphism group $G:=\operatorname{Aut}(\mathfrak{A})$. The partition $\mathcal{B}=\left(B_{\alpha}: \alpha \in \kappa\right)$ of $A$ distinguishes the relational structure $\mathfrak{A}$ if

$$
G \downarrow \mathcal{B}:=\left\{g \in G: \forall \alpha \in \kappa g\left(B_{\alpha}\right)=B_{\alpha}\right\}
$$

contains as its only element the identity automorphism of $\mathfrak{A}$. Here and elsewhere when $B$ is a subset of the domain of a function $g$, then $g(B)$ means the setwise mapping of its elements $\{g(b): b \in B\}$. The distinguishing number of $\mathfrak{A}$, written $D(\mathfrak{A})$, is the smallest cardinality of the set of blocks of a distinguishing partition of $A$.

This is more accurately a property of the group $G$ acting on the set $A$, and for that reason we will often refer to this number as the distinguishing number of $G$ acting on $A$.

The skeleton of a structure $\mathfrak{A}$ is the set of finite induced substructures of $\mathfrak{A}$ and the age of $\mathfrak{A}$ consists of all relational structures isomorphic to an element of the skeleton of 
$\mathfrak{A}$. The boundary of $\mathfrak{A}$ consists of finite relational structures with the same signature as $\mathfrak{A}$ which are not in the age of $\mathfrak{A}$ but for which every strictly smaller induced substructure is in the age of $\mathfrak{A}$.

A local isomorphism of $\mathfrak{A}$ is an isomorphism between two elements of the skeleton of $\mathfrak{A}$. The relational structure $\mathfrak{A}=(A, \mathbf{R})$ is homogeneous if every local isomorphism of $\mathfrak{A}$ has an extension to an automorphism of $\mathfrak{A}$.

Definition 1.1. A class $\mathcal{A}$ of structures has amalgamation if for any three elements $\mathfrak{B}_{0}$ and $\mathfrak{B}_{1}$ and $\mathfrak{C}$ of $\mathcal{A}$ and all embeddings $f_{0}$ of $\mathfrak{C}$ into $\mathfrak{B}_{0}$ and $f_{1}$ of $\mathfrak{C}$ into $\mathfrak{B}_{1}$ there exists a structure $\mathfrak{D}$ in $\mathcal{A}$ and embeddings $g_{0}$ of $\mathfrak{B}_{0}$ into $\mathfrak{D}$ and $g_{1}$ of $\mathfrak{B}_{1}$ into $\mathfrak{D}$ so that $g_{0} \circ f_{0}=g_{1} \circ f_{1}$.

The relational structure $\mathfrak{A}=(A, \mathbf{R})$ has amalgamation if its age has amalgamation.

A powerful characterization of countable homogeneous structures was established by Fraïssé.

Theorem 1.2. [4, 5] A countable structure is homogeneous if and only if its age has amalgamation.

Moreover a countable relational structure $\mathfrak{A}=(A, \mathbf{R})$ is homogeneous if and only if it satisfies the following mapping extension property: If $\mathfrak{B}=(B, \mathbf{R})$ is an element of the age of $\mathfrak{A}$ for which the substructure of $\mathfrak{A}$ induced on $A \cap B$ is equal to the substructure of $\mathfrak{B}$ induced on $A \cap B$, then there exists an embedding of $\mathfrak{B}$ into $\mathfrak{A}$ which is the identity on $A \cap B$.

Finally, given a class $\mathcal{A}$ of finite structures closed under isomorphism, substructures, joint embeddings (any two members of $\mathcal{A}$ embed in a third), and which has amalgamation, then there is a countable homogeneous structure whose age is $\mathcal{A}$.

A stronger notion is that of free amalgamation. Before we define this notion, we need the concept of adjacent elements in a relational structure.

Given a relational structure $\mathfrak{A}=(A, \mathbf{R})$, the elements $a, b \in A$ are called adjacent if there exists a sequence $\left(s_{0}, s_{1}, s_{2}, \ldots, s_{n-1}\right)$ of elements of $A$ with $s_{i}=a$ and $s_{j}=b$ for some $i \neq j \in n$ and a relation $R \in \mathbf{R}$ so that $R\left(s_{0}, s_{1}, s_{2}, \ldots, s_{n-1}\right)$. A relational structure is complete if $a$ and $b$ are adjacent for all distinct elements $a$ and $b$ of the structure.

Definition 1.3. Let $\mathfrak{A}=(A, \mathbf{R})$ be a relational structure and $\mathfrak{B}_{0}=\left(B_{0}, \mathbf{R}\right), \mathfrak{B}_{1}=\left(B_{1}, \mathbf{R}\right)$ two elements in the age of $\mathfrak{A}$. The relational structure $\mathfrak{D}=(D, \mathbf{R})$ is a free amalgam of $\mathfrak{B}_{0}$ and $\mathfrak{B}_{1}$ if:

1. $D=B_{0} \cup B_{1}$.

2. The substructure on $B_{0}$ induced by $\mathfrak{D}$ is $\mathfrak{B}_{0}$.

3. The substructure on $B_{1}$ induced by $\mathfrak{D}$ is $\mathfrak{B}_{1}$.

4. If $a \in B_{0} \backslash B_{1}$ and $b \in B_{1} \backslash B_{0}$ then $a$ and $b$ are not adjacent in $\mathfrak{D}$. 
The relational structure $\mathfrak{A}$ has free amalgamation if every two elements of its age have a free amalgam.

Note that if a relational structure has free amalgamation then it has amalgamation.

The following, due to N. Sauer, characterizes countable homogeneous structures with free amalgamation as those whose boundary consists of finite complete structures.

Theorem 1.4. [11] If $\mathcal{C}$ is a countable set of finite complete relational structures having the same signature then there exists a unique countable homogeneous structure $\mathfrak{A}$ whose boundary is $\mathcal{C}$, and has free amalgamation.

Conversely, if $\mathfrak{A}$ is a countable homogeneous structure with free amalgamation, then the boundary of $\mathfrak{A}$ consists of finite complete structures.

The article is organized as follows. We will see that surprisingly many homogeneous structures have distinguishing number 2 , and the main tool in demonstrating these results is developed in section 2. We use it immediately in section 3 on countable homogeneous structures with free amalgamation and minimal arity two. In section 4, we compute the distinguishing number of all countable homogeneous undirected graphs, and we do the same in section 5 for all countable homogeneous directed graphs.

\section{Permutation groups and fixing types}

In this section we develop a powerful sufficient condition for a permutation group acting on a set to have distinguishing number 2, which we will use on a variety of homogeneous relational structures in subsequent sections.

Let $G$ be a permutation group acting on the set $A$. For $F \subseteq A$, we write $G_{\{F\}}:=$ $\{g \in G: g(F)=F\}$ and $G_{(F)}:=\{g \in G: \forall x \in F g(x)=x\}$. We define equivalence relations $a \stackrel{\{F\}}{\sim} b$ if there exists $g \in G_{\{F\}}$ with $g(a)=b$, and $a \stackrel{(F)}{\sim} b$ if there is $g \in G_{(F)}$ with $g(a)=b$. We write $\neg(a \stackrel{(F)}{\sim} b)$ if it is not the case that $a \stackrel{(F)}{\sim} b$. Note that if $F_{1} \subseteq F_{2}$ and $\neg\left(a \stackrel{\left(F_{1}\right)}{\sim} b\right)$ then $\neg\left(a \stackrel{\left(F_{2}\right)}{\sim} b\right)$.

We call the pair $(F, T)$ a type (on $G$ ), if $F \subseteq A$ is finite and $T$ is a non empty equivalence class of $\stackrel{(F)}{\sim}$ disjoint from $F$. The pair $(F, T)$ is a set type if $F \subseteq A$ is finite and $T$ is a non empty equivalence class of $\stackrel{\{F\}}{\sim}$ disjoint from $F$. The pair $(F, T)$ is an extended set type if there exists a set $\mathcal{T}$ of subsets of $A$ so that for every $S \in \mathcal{T}$ the pair $(F, S)$ is a set type and $T=\bigcup_{S \in \mathcal{T}} S$.

Note that if $(F, T)$ is a type then $(g(F), g(T))$ is a type for all $g \in G$, and if $(F, T)$ is a set type then $(g(F), g(T))$ is a set type for all $g \in G$. Hence if $(F, T)$ is an extended set type then $(g(F), g(T))$ is an extended set type for all $g \in G$.

Lemma 2.1. Let $(F, T)$ be a set type. Then $g(T)=T$ for every $g \in G_{\{F\}}$. If $h$ and $k$ are elements of $G$ with $h(F)=k(F)$ then $h(T)=k(T)$. 
Proof. Let $g \in G_{\{F\}}$. Then clearly $g(T) \subseteq T$ and since $g^{-1} \in G_{\{F\}}$, then $\left(g^{-1}\right)(T) \subseteq T$ as well implying that $g(T)=T$. For $h, k \in G$ with $h(F)=k(F)$, then $k^{-1} \circ h \in G_{\{F\}}$ implying that $\left(k^{-1}\right)(h(T))=T$ and therefore $h(T)=k(T)$.

Corollary 2.2. Let $(F, T)$ be an extended set type. Then $g(T)=T$ for every $g \in G_{\{F\}}$. If $h$ and $k$ are elements of $G$ with $h(F)=k(F)$ then $h(T)=k(T)$.

Definition 2.3. An extended set type $(F, T)$ has the cover property if for every finite subset $H$ of $G \backslash G_{\{F\}}$ the set

$$
T \backslash \bigcup_{h \in H} h(T)
$$

is infinite.

Note that if a set type $(F, T)$ has the cover property then $(g(F), g(T))$ has the cover property for every $g \in G$.

Lemma 2.4. Let $(F, T)$ be an extended set type with the cover property. Let $B$ be a finite subset of $A$ with $F \nsubseteq B$. Then the set

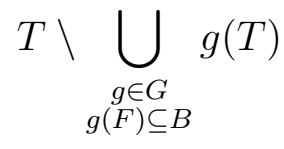

is infinite.

Proof. For $g \in G$ let $g\lceil F$ be the restriction of $g$ to $F$. The set $K$ of functions $g\lceil F$ with $g(F) \subseteq B$ is finite. For every function $k \in K$ let $\bar{k}$ be an extension of $k$ to an element of $G$. Then $H=\{\bar{k}: k \in K\}$ is finite, and it follows from Corollary 2.2 that:

$$
\bigcup_{\substack{g \in G \\ g(F) \subseteq B}} g(T)=\bigcup_{\bar{k} \in H} \bar{k}(T) .
$$

But the cover property implies that the set

$$
T \backslash \bigcup_{\bar{k} \in H} \bar{k}(T)
$$

is infinite, completing the proof.

Corollary 2.5. Let $(F, T)$ be an extended set type which has the cover property. Let $B$ be a finite subset of $A$ and $h \in G$ such that $h(F) \nsubseteq B$. Then the set

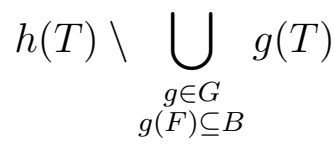

is infinite. 
Proof. The pair $(h(F), h(T))$ is again an extended set type with the cover property. Now observe that $g(F) \subseteq B$ if and only if $\left(g \circ h^{-1}\right)(h(F)) \subseteq B$.

The existence of the following special kind of extended set type will suffice to guarantee a small distinguishing number.

Definition 2.6. The pair $(F, T)$ is a fixing type for the permutation group $G$ acting on $A$ if there is a partition $A=\left(A_{i}: i<2\right)$ such that:

1. For every element $g \in G$ and finite $S \subseteq A_{0}$ such that $g(S) \subseteq A_{0}$, there is a $g_{0} \in G_{0}=G_{\left\{A_{0}\right\}}$ such that $g\left\lceil S=g_{0} \uparrow S\right.$.

2. $(F, T)$ is an extended set type of $G_{0}$ acting on $A_{0}$, and $(F, T)$ has the cover property.

3. For all $b \in T$ there exists $a \in F$ and $g \in G$ (equivalently $g \in G_{0}$ ) with $g(F)=$ $(F \backslash\{a\}) \cup\{b\}$.

4. $\neg(a \stackrel{(T)}{\sim} b)$ for all $a, b \in A \backslash(T \cup F)$ with $a \neq b$.

5. $\neg(a \stackrel{(A \backslash F)}{\sim} b)$ for all $a, b \in F$ with $a \neq b$.

Note that if $(F, T)$ is a fixing type and $g \in G_{0}$, then $(g(F), g(T))$ is again a fixing type for the same partition. Note also that if $F$ is a singleton as will often be the case in the present paper, then item 5 is vacuous. We simply write $(a, T)$ when $F$ is the singleton $\{a\}$. Item 3 is guaranteed by a transitive group action such as the automorphism group of a homogeneous relational structure as will also be the case here. Moreover almost all applications will only require a trivial partition $A=\left(A_{i}: i<2\right)$ where $A=A_{0}\left(A_{1}=\emptyset\right)$, in which case item 1 is trivially true. All this to say that often only items 2 and 4 need to be verified. Nevertheless the full generality will be used in a few crucial cases.

Example 2.7. The Rado graph is the amalgamation of all finite undirected graphs. The Rado graph is therefore homogeneous by Theorem 1.2 and is often called the random graph (it can be described by randomly selecting edges between pairs of vertices). If $V$ denotes the set of vertices and $v \in V$, let $T$ be the set of vertices which are adjacent to $v$. Then $(v, T)$ is a fixing type of the automorphism group of the Rado graph acting on $V$ using the trivial partition $V=\left(V_{0}\right)$.

Example 2.8. Consider the amalgamation of all finite three uniform hypergraphs, called the universal three uniform hypergraph. Let $V$ be its set of vertices, $\{u, v, w\}$ be a hyperedge of the hypergraph, and $T$ be the set of elements $x \in V \backslash\{u, v, w\}$ for which $\{x, u, v\}$, $\{x, v, w\}$ and $\{x, u, w\}$ are all hyperedges. Then $(\{u, v, w\}, T)$ is a fixing type of the automorphism group of the universal three uniform hypergraph acting on $V$, again using the trivial partition $V=\left(V_{0}\right)$.

We now come to the main result of this section, which will allow us to show that many structures have distinguishing number two. 
Theorem 2.9. Let $G$ be a permutation group acting on the countable set $A$. If there exists a fixing type for the action of $G$ on $A$ then the distinguishing number of $G$ acting on $A$ is two.

Proof. Let $(F, T)$ be a fixing type for the action of $G$ on $A$ with corresponding partition $A=\left(A_{i}: i<2\right)$. Let $\left(b_{i}: i \in \omega\right)$ be an $\omega$-enumeration of $T$ and for every $i \in \omega$, use item 3 of Definition 2.6 to produce $a_{i} \in F$ and $g_{i} \in G$ such that $g_{i}(F)=\left(F \backslash\left\{a_{i}\right\}\right) \cup\left\{b_{i}\right\}:=F_{i}$. By item 1 , we may assume that $g_{i} \in G_{0}$, so let $T_{i}:=g_{i}(T) \subseteq A_{0}$. It follows that $\left(F_{i}, T_{i}\right)$ is a fixing type for every $i \in \omega$ and the same partition of $A$.

We construct a sequence $\left(S_{i}: i \in \omega\right)$ of finite subsets of $A_{0}$ so that for every $i \in \omega$ :

a. $S_{i} \cap T=\emptyset$.

b. $S_{i} \subseteq T_{i}$.

c. $\left|S_{i}\right|=1+\sum_{j \in i}\left|S_{j}\right|$.

d. $S_{i} \cap g(T)=\emptyset$ for every $g \in G$ such that $g(F) \subseteq C_{i}:=F \cup\left\{b_{j}: j \in i\right\} \cup \bigcup_{j<i} S_{j}$.

Notice that item d. implies that $S_{j} \cap S_{i}=\emptyset$ for all $j<i$ since $S_{j} \subseteq T_{j}=g_{j}(T)$ and $g_{j}(F) \subseteq C_{i}$.

The construction proceeds by induction. Assume $S_{i-1}$ has been constructed. Now $g_{i}(F) \nsubseteq$ $C_{i}$ since $b_{i}$ belongs to the former and not the latter. Since $(F, T)$ is an extended set type of $G_{0}$ acting on $A_{0}$, and $(F, T)$ has the cover property, Corollary 2.5 therefore shows that

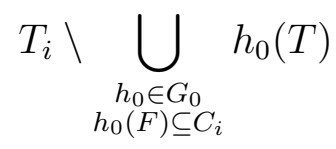

is infinite. However if $g \in G$ is such that $g(F) \subseteq C_{i}$, then consider any $b=g(a) \in$ $g(T) \cap A_{0}$. By item 1 of Definition 2.6, there is $h_{0} \in G_{0}$ such that $h_{0}(F)=g(F) \subseteq C_{i}$ and $h_{0}(a)=g(a)=b$. Therefore $b \in h_{0}(T)$, i.e. $g(T) \cap A_{0} \subseteq h_{0}(T)$. Hence we conclude that

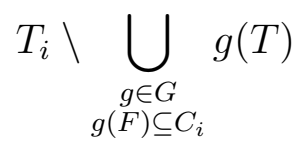

is infinite, allowing us to obtain $S_{i}$ as desired. This completes the construction.

Let $S=\bigcup_{i \in \omega} S_{i}$ and $\mathcal{B}=\left(B_{0}, B_{1}\right)$ be the partition of $A$ with $B_{0}:=F \cup T \cup S \subseteq A_{0}$, and fix $g \in G \downarrow \mathcal{B}$. It suffices to show that $g$ is the identity, and this will result from the following four claims.

Claim 1. $g(F)=F$.

Proof. We begin by the following.

Subclaim 1. $T \backslash g(T)$ is finite. 
Proof. For any $h \in G \downarrow \mathcal{B}, h(F)$ is a subset of $B_{0} \subseteq A_{0}$ and hence a subset of $C_{i}$ for some $i \in \omega$. But this means by item d. that $S_{j} \cap h(T)=\emptyset$ for all $j \geqslant i$. Since $h\left(B_{0}\right)=B_{0}$, this means $h(T) \subseteq F \cup T \cup \bigcup_{k<i} S_{k}$, and therefore $h(T) \backslash T$ is finite.

Since $g^{-1} \in G \downarrow \mathcal{B}$, we conclude that $g^{-1}(T) \backslash T$ is finite, and therefore $T \backslash g(T)$ is finite.

Assume now for a contradiction that $g(F) \neq F$. Then by item 1 of Definition 2.6 there is $g_{0} \in G_{0}$ such that $g_{0}(F)=g(F) \neq F$.

Subclaim 2. $g(T) \subseteq g_{0}(T)$.

Proof. Let $b \in T$. Then $c=g(b) \in B_{0} \subseteq A_{0}$, and by item 1 of Definition 2.6, there is $g_{1} \in G_{0}$ such that $g_{1}(F)=g_{0}(F)$ and $g_{1}(b)=g(b)=c$. But then $g_{1}(T)=g_{0}(T)$ by Corollary 2.2, so $c=g(b)=g_{1}(b) \in g_{0}(T)$.

But $T \backslash g_{0}(T)$ is infinite since $(F, T)$ is an extended set type of $G_{0}$ acting on $A_{0}$ and has the cover property, and therefore $T \backslash g(T)$ is infinite by Subclaim 2. This contradicts Subclaim 1 and completes the proof of Claim 1.

Claim 2. $g(x)=x$ for every element $x \in T$.

Proof. We first verify that $g(T)=T$. Indeed let $b=g(a)$ for some $a \in T$. Since $b \in A_{0}$, then by item 1 of Definition 2.6 choose $g_{0} \in G_{0}$ such that $g_{0}(F)=g(F)=F$ and $g_{0}(a)=b=g(a)$. But $g_{0}(T)=T$ by Corollary 2.2, so $b \in T$, and therefore $g(T) \subseteq T$. Similarly $g^{-1}(T) \subseteq T$ since $g^{-1}(F)=F$ as well, and therefore $T \subseteq g(T)$.

Now if $g\left(b_{i}\right)=b_{k}$ with $i>k$ then $g \circ g_{i}(F)=g\left(F_{i}\right) \subseteq F \cup\left\{b_{k}\right\} \subseteq C_{i}$. It follows from item d. that $g\left(T_{i}\right) \cap S_{j}=\emptyset$ for all $j \geqslant i$, and hence $g\left(S_{i}\right) \cap S_{j}=\emptyset$ for all $j \geqslant i$. On the other hand $g(S)=S$ because $g(T \cup F)=T \cup F$ as proved above. We conclude that $g\left(S_{i}\right) \subseteq \bigcup_{j \in i} S_{j}$, violating item c.

Hence $g$ induces an $\leqslant$-order preserving map of $\omega$ onto $\omega$ which implies $g\left\lceil T=\mathrm{id}_{T}\right.$.

Claim 3. $g(x)=x$ for every element $x \in A \backslash(T \cup F)$.

Proof. Follows from item 4 of Definition 2.6.

Claim 4. $g(x)=x$ for every element $x \in F$.

Proof. Follows from item 5 of Definition 2.6.

This completes the proof of Theorem 2.9. 


\section{Homogeneous relational structure with free amal- gamation}

Several countable homogeneous structure do have free amalgamation. These include the Rado Graph and universal three uniform hypergraphs which we have seen already, but also several other homogeneous structures including the universal $K_{n}$-free homogeneous graphs. For these structures, the distinguishing number is as low as it can be.

Theorem 3.1. Let $\mathfrak{A}=(A, \mathbf{R})$ be a countable homogeneous structure with signature $\mu$, minimal arity at least two, and having free amalgamation. Then the distinguishing number of $\mathfrak{A}$ is two.

Proof. Let $G=\operatorname{Aut}(\mathfrak{A})$. We have to prove that the distinguishing number of the permutation group $G$ acting on the countable set $A$ is two.

Let $n \in \omega$ be the smallest arity of a relation in $\mathbf{R}$ and let $\mathbf{P} \subseteq \mathbf{R}$ be the set of relations in $\mathbf{R}$ having arity $n$. Let $F \subseteq A$ have cardinality $n-1$ and let $T$ be the set of all $b \in A$ for which there exists a sequence $\vec{s}$ with entries in $F \cup\{b\}$ and $R \in \mathbf{P}$ with $R(\vec{s})$.

The pair $(F, T)$ is an extended set type, and it follows from Theorem 2.9 that if $(F, T)$ is a fixing type for the permutation group $G$ acting on $A$ then the distinguishing number of $\mathfrak{A}$ is two.

We verify the items of Definition 2.6 using the trivial partition $A=\left(A_{0}\right)$.

Item 2: Let $H$ be a finite subset of $G$ so that $F \neq h(F)$ for all $h \in H$. Let

$$
B:=\left(\bigcup_{h \in H} h(F)\right) \backslash F \text { and } \mathfrak{B} \text { the substructure of } \mathfrak{A} \text { induced by } F \cup B \text {. }
$$

Let $x$ be an element not in $A$ and $R \in \mathbf{P}$ and $\mathfrak{X}=(F \cup\{x\}, \mathbf{R})$ be a relational structure with signature $\mu$ in which $R(\vec{s})$ for some tuple $\vec{s}$ with entries in $F \cup\{x\}$ so that $\mathfrak{X}$ is an element in the age of $\mathfrak{A}$. Let $\mathfrak{C}$ be the free amalgam of $\mathfrak{X}$ with $\mathfrak{B}$. It follows from the mapping extension property of $\mathfrak{A}$ that there exists a type $(F \cup B, U)$ so that $u \in T \backslash h(T)$ for every element $u \in U$ and $h \in H$. Item 2 follows because $U$ is infinite.

Item 3: Because $n \geqslant 2$ there exists an element $a \in F$. The sets $F$ and $(F \backslash\{a\}) \cup\{b\}$ have cardinality $n-1$ and the minimal cardinality of $\mathfrak{A}$ is $n$. Hence every bijection of $F$ to $(F \backslash\{a\}) \cup\{b\}$ is a local isomorphism, and by homogeneity extends top a full automorphism of $\mathfrak{A}$.

Item 4: Let $a, b \in A \backslash(T \cup F)$ with $a \neq b$. Let $R \in \mathbf{P}$. Let $E$ with $|E|=n-1$ be a set of elements not in $A$ and $\mathfrak{X}=(F \cup E, \mathbf{R})$ a relational structure in the age of $A$ so that there is an embedding of $\mathfrak{X}$ into $\mathfrak{A}$ which fixes $F$ and maps $E$ into $T$. Let $\mathfrak{Y}=(E \cup\{a\}, \mathbf{R})$ be a relational structure in the age of $\mathfrak{A}$ so that $R(\vec{s})$ for some tuple $\vec{s}$ with entries in $E \cup\{a\}$. Let $\mathfrak{B}$ be the free amalgam of $\mathfrak{X}$ and $\mathfrak{Y}$. Note that the restriction of $\mathfrak{B}$ to $F \cup\{a\}$ is equal to the restriction of $\mathfrak{A}$ to $F \cup\{a\}$, for otherwise $a \in T$.

Now let $\mathfrak{Z}=(F \cup\{a, b\}, \mathbf{R})$ be the substructure of $\mathfrak{A}$ induced by $F \cup\{a, b\}$ and let $\mathfrak{C}$ be the free amalgam of $\mathfrak{Z}$ and $\mathfrak{B}$. The substructure of $\mathfrak{C}$ induced by $F \cup\{a, b\}$ is again equal 
to the substructure of $\mathfrak{A}$ induced by $F \cup\{a, b\}$. Hence there exists an embedding $f$ of $\mathfrak{C}$ into $\mathfrak{A}$ which fixes $F \cup\{a, b\}$. It follows from the construction of $\mathfrak{C}$ that $f(E) \subseteq T$. Then $\neg(a \stackrel{(T)}{\sim} b)$ because $\neg(a \stackrel{(f(E))}{\sim} b)$.

Item 5: Let $a \neq b \in F$ and $E$ be a set of elements not in $A$ with $|E|=n-1$. Let $\mathfrak{X}=(E \cup\{a\}, \mathbf{R})$ be an element in the age of $\mathfrak{A}$ so that $R(\vec{s})$ for some $R \in \mathbf{P}$ and some tuple $\vec{s}$ of elements in $E \cup\{a\}$. Let $\mathfrak{Y}=(\{a, b\}, \mathbf{R})$ be the substructure of $\mathfrak{A}$ induced by $F$. Let $\mathfrak{B}$ be the free amalgam of $\mathfrak{X}$ and $\mathfrak{Y}$. There exists an embedding $f$ of $\mathfrak{B}$ into $\mathfrak{A}$ which fixes $F$. Then $\neg(a \stackrel{(A \backslash F)}{\sim} b)$ because $\neg(a \stackrel{(f(E))}{\sim} b)$.

\section{Homogeneous undirected graphs}

\subsection{Finite homogeneous undirected graphs}

The finite homogeneous graphs were classified by Tony Gardiner [G]. Moreover the distinguishing number of finite graphs in general has been extensively studied, see for example the work of Imrich and Klavžar in [8] for references. In particular the five cycle is homogeneous and $D\left(C_{5}\right)=3$ as we have already mentioned. If $K_{n}$ denotes the complete graph on $n$ vertices, then clearly $D\left(K_{n}\right)=D\left(K_{n}^{c}\right)=n$.

More interestingly we have the following regarding the family of finite homogeneous graphs $m \cdot K_{n}$ consisting of $m$ copies of $K_{n}$ for any $m, n \in \mathbb{N}$.

Theorem 4.1. For $m, n \in \mathbb{N}$, then $D\left(m \cdot K_{n}\right)=D\left(\left(m \cdot K_{n}\right)^{c}\right)$ is the least $k \in \mathbb{N}$ such that $\left(\begin{array}{c}k \\ n\end{array}\right) \geqslant m$.

Proof. The distinguishing number of a graph equals that of its complement, so we concentrate on $m \cdot K_{n}$.

Each copy of $K_{n}$ requires $n$ distinct labels, and any two copies of $K_{n}$ must receive different sets of $n$ distinct labels to avoid a nontrivial automorphism. It is clearly a sufficient condition, so we must therefore find $m$ different sets of $n$ distinct labels.

The last finite homogeneous undirected graph is the line graph of $K_{3,3}$, which is isomorphic to its complement. Its distinguishing number is proved in [8] to be 3 , but for completeness we supply a direct proof.

Theorem 4.2. $[8] D\left(L\left(K_{3,3}\right)\right)=3$.

Proof. One must first show that $D\left(L\left(K_{3,3}\right)\right)>2$. However one can observe that a finite homogeneous structure has distinguishing number 2 exactly if it can be partitioned into two rigid (no nontrivial automorphisms) induced substructures. But $L\left(K_{3,3}\right)$ has 9 vertices, and one verifies that there are no rigid graphs with at most 4 (even 5 ) vertices.

A distinguishing 3-labeling of $L\left(K_{3,3}\right)$ can be obtained as follows. Let $K_{3,3}$ be the complete bipartite graph for the two sets of vertices $\{a, b, c\}$ and $\{x, y, z\}$. Then label the edge $(a, x)$ with the first label, the two edges $(a, y)$ and $(b, z)$ with the second label, and 
all other edges with a third label. Then one verifies that only the identity automorphism of $L\left(K_{3,3}\right)$ preserves these labels.

\subsection{Countable homogeneous undirected graphs}

The countably infinite homogeneous undirected graphs have been classified by Alistair Lachlan and Robert Woodrow in [10].

The first class consists of graphs of the form $m \cdot K_{n}$ for $m+n=\omega$ and their complement, all easily seen to have distinguishing number $\omega$. We have already seen that, proved in [9], the distinguishing number of the Rado graph is 2 . Then for each $n \geqslant 3$ we find the generic graph which is the amalgamation of all finite graphs omitting the $n$-clique $K_{n}$. These graphs have free amalgamation by the characterization of Theorem 1.4, and therefore all of them and their complements have distinguishing number two by Theorem 3.1. For $n=2$, the generic graph omitting $K_{2}$ is simply an infinite antichain $I_{\infty}$ and has, like its complement $K_{\omega}$, distinguishing number $\omega$.

\section{$5 \quad$ Homogeneous directed graphs}

We follow Gregory Cherlin's catalog of homogeneous directed graph, see [2] and [3], and in each case compute their distinguishing number.

\subsection{Deficient graphs}

The deficient structures are those omitting a 2-type, meaning a structure on 2 elements. In the case of graphs they are the $n$-antichain $I_{n}$ omitting an edge, clearly having distinguishing number $n$ respectively, and the tournaments omitting $I_{2}$.

The four remaining homogeneous tournaments (beside $I_{1}$ ) are as follows (see [3]). The

first is the oriented 3 -cycle $\vec{C}_{3}$, which was already seen to have distinguishing number 2. Next is the rational numbers $\mathbb{Q}$ viewed as a directed graph with edges following the standard ordering, which can easily seen to have distinguishing number $\omega$. Indeed consider a labeling of $\mathbb{Q}$ into finitely many labels. Then we can find an interval $I$ either contained in one of the labels, or else on which each label is either dense or empty. Then a back and forth argument, leaving $\mathbb{Q} \backslash I$ intact but moving $I$, produces a non-trivial automorphism.

In preparation to handle the last two homogeneous tournaments, we say (following Cherlin [3]) that a vertex $a$ dominates a vertex $b$ if the edge between them is oriented toward $b$, and write ' $a$ and $a^{\prime}$ for the sets of vertices dominating and dominated by $a$, respectively. We shall also later use $a^{\perp}$ to denote the set of vertices not connected to $a$. A tournament is called a local order if for every vertex $a$, the induced tournaments on $a^{\prime}$ and ' $a$ are linear orders. The class of finite local orders is an amalgamation class and the corresponding homogeneous tournament is called the dense local order, written $\mathbb{Q}^{*}$.

We can now prove the following.

Theorem 5.1. $D\left(\mathbb{Q}^{*}\right)=\omega$. 
Proof. $\mathbb{Q}^{*}$ can be realized by partitioning $\mathbb{Q}$ into two disjoint dense sets $Q_{0}$ and $Q_{1}$, and reversing the direction of edges from one of these sets to the other.

Consider now a labeling of $\mathbb{Q}^{*}$ into finitely many labels. Then one can find an interval $I$ of the rationals $\mathbb{Q}$ such that restricted to each $Q_{i}$ it is either contained in one of the labels, or else on which each label is either dense or empty. Then a back and forth argument, leaving $\mathbb{Q} \backslash I$ intact but moving $I$, produces a non-trivial automorphism.

The last countable homogeneous tournament is the universal tournament $\mathbb{T}^{\infty}$, corresponding to the amalgamation of all finite tournaments.

Theorem 5.2. $D\left(\mathbb{T}^{\infty}\right)=2$.

Proof. Let $G$ be the automorphism group of $\mathbb{T}^{\infty}=\left(T^{\infty}, E\right)$. Fix $a \in T^{\infty}$ and let $T=a^{\prime}$ be the set of all elements of $T^{\infty}$ dominated by $a$. We will show that $(a, T)$ is a fixing type of $G$ acting on $T^{\infty}$ with trivial partition. As noted after after Definition 2.6, only items 2 and 4 need attention in this case.

Item 2: $(a, T)$ is easily seen to be an extended set type. For the cover property, we have to prove that if $S$ is a finite subset of $T^{\infty} \backslash\{a\}$ then there are infinitely many elements $b \in T$ dominating each $s \in S$.

Let $x$ be an element not in $T^{\infty}$ and $\mathfrak{X}=(S \cup\{a, x\}, E)$ the tournament so that $\mathfrak{X}$ restricted to $S \cup\{a\}$ is equal to $\mathbb{T}^{\infty}$ restricted to $S \cup\{a\}$, and $x$ dominates every $s \in S$, and $a$ dominates $x$. By the mapping extension property, there are infinitely many embeddings of $\mathfrak{X}$ into $\mathbb{T}^{\infty}$ which fix $S$.

Item 4: Let $b, c \in T^{\infty} \backslash(T \cup\{a\})$ with $b \neq c$. Both $b$ and $c$ must dominate $a$. Let $x$ be an element not in $T^{\infty}$ and $\mathfrak{X}=(\{a, b, c, x\}, E)$ the tournament so that the restriction of $\mathfrak{X}$ to $\{a, b, c\}$ is equal to the restriction of $\mathfrak{T}^{\infty}$ to $\{a, b, c\}, a$ and $b$ dominate $x$ and $x$ dominates $c$. By the mapping extension property, there is an embedding of $\mathfrak{X}$ which fixes $\{a, b, c\}$ and maps $x$ into $T$. Then $\neg(b \stackrel{(T)}{\sim} c)$.

Item 5: This condition is vacuous since $F$ has only one element.

\section{$5.2 \quad$ Imprimitive graphs}

A graph (and more generally a relational structure) is imprimitive if it carries a nontrivial 0-definable equivalence relation, that is an equivalence relation definable from a formula in the given relation language without extra distinguished parameters. In the homogeneous case, such an equivalence relation must be the union of equality with either incomparability relation or its complement. A graph is called primitive otherwise.

The first occurrence of these kinds of imprimitive graphs happens when the graph is the wreath product $H_{1}\left[H_{2}\right]$ of two graphs $H_{1}$ and $H_{2}$ having no 2-types in common, obtained by replacing each vertex of $H_{1}$ by a copy of $H_{2}$. In this case they are of the form $H\left[I_{n}\right]$ or $I_{n}[H]$ for $1<n<\infty$ and $H$ one of the four non-degenerate tournaments listed above. 
It is not hard to compute that $D\left(H\left[I_{n}\right]\right)$ is the least integer $k$ such that $\left(\begin{array}{c}k \\ n\end{array}\right) \geqslant D(H)$. In particular $D\left(\vec{C}_{3}\left[I_{n}\right]\right)=D\left(\mathbb{T}^{\infty}\left[I_{n}\right]\right)=n+1$ for $n>1$, and $D\left(\mathbb{Q}\left[I_{n}\right]\right)=D\left(\mathbb{Q}^{*}\left[I_{n}\right]\right)=\omega$. Similarly $D\left(I_{n}\left[\vec{C}_{3}\right]\right)=D\left(I_{n}\left[\mathbb{T}^{\infty}\right]\right)$ is the least integer $k$ such that $2\left(\begin{array}{c}k \\ 2\end{array}\right) \geqslant n$. Clearly $D\left(I_{n}[\mathbb{Q}]\right)=D\left(I_{n}\left[\mathbb{Q}^{*}\right]\right)=\omega$.

Another family of homogeneous graphs is obtained from a tournament $H$ as follows. First consider the new directed graph $H^{+}=H \cup\{v\}$ where $H \subseteq v^{\prime}$. Then form $\widehat{H}$ as the union of two copies $H_{1}^{+}$and $H_{2}^{+}$of $H^{+}$, and for $u_{1} \in H_{1}^{+}$and $v_{2} \in H_{2}^{+}$corresponding to $u, v \in H^{+}$, put an edge from $u_{1}$ to $v_{2}$ exactly if there is one from $v$ to $u$ in $H^{+}$(reversed). Clearly $\widehat{I}_{1}=\vec{C}_{4}$, the directed 4-cycle, and therefore $D\left(\widehat{I_{1}}\right)=2$ (recall the undirected 4cycle $C_{4}$ has distinguishing number 3$)$. One can also show that $D\left(\widehat{\overrightarrow{C_{3}}}\right)=2$. Indeed, label the vertices of each of the two copies of $\vec{C}_{3}$ in the same fashion with the same two labels, then label the two new vertices differently but again using the same two labels. Then these new vertices are fixed since they are the only ones not related (perpendicular) to vertices of a different label. From this one verifies that all other vertices are also fixed.

For the infinite graphs $\widehat{\mathbb{Q}}$ and $\widehat{\mathbb{T}^{\infty}}$, the extra vertices are not really needed. An argument similar to that of Theorem 5.1 shows that $D(\widehat{\mathbb{Q}})=\omega$. It is interesting that $\widehat{\mathbb{Q}^{*}}$ itself is not homogeneous (see [3]).

Finally, the proof of Theorem 5.2 can be adapted to show that $D\left(\widehat{\mathbb{T}^{\infty}}\right)=2$.

Theorem 5.3. $D\left(\widehat{\mathbb{T}^{\infty}}\right)=2$.

Proof. Let $\widehat{T^{\infty}}$ be the domain of $\widehat{T^{\infty}}$. The fixing type used in the proof of Theorem 5.2 shows that it is a fixing type $(a, T)$ for $G$ acting on $\widehat{T^{\infty}}$ using the partition $\widehat{T^{\infty}}=\left(A_{0}, A_{1}\right)$, where $A_{0}$ is a copy of $\mathbb{T}^{\infty}$.

Indeed the homogeneity of $\mathbb{T}^{\infty}$ shows that it satisfies item 1 . Item 2 follows from the proof of Theorem 5.2, and items 3 and 5 are immediate.

Item 4 follows by considering the necessary cases. Of course the cases where $b, c \in A_{0}$ follow from the proof of Theorem 5.2. As an example consider $b_{1}, c_{1} \in A_{1}$, both dominating $a$. Then by construction they correspond to $b_{0}, c_{0} \in A_{0}$, both dominated by $a$ (and therefore in $T)$. Let $x$ be an element not in $T^{\infty}$ and $\mathfrak{X}=\left(\left\{a, b_{0}, c_{0}, x\right\}, R\right)$ the tournament so that the restriction of $\mathfrak{X}$ to $\left\{a, b_{0}, c_{0}\right\}$ is equal to the restriction of $\mathbb{T}^{\infty}$ to $\left\{a, b_{0}, c_{0}\right\}$, $a$ and $b_{0}$ dominate $x$ and $x$ dominates $c^{\prime}$. By the mapping extension property, there is an embedding of $\mathfrak{X}$ which fixes $\left\{a, b_{0}, c_{0}\right\}$ and maps $x$ into $T$. Then $\neg\left(b_{0} \stackrel{(T)}{\sim} c_{0}\right)$, and it follows that $\neg(b \stackrel{(T)}{\sim} c)$. The other cases are similar.

Call two vertices $a$ and $b$ perpendicular, written $\perp$, if they have no edges between them. The graph $n * I_{\infty}$ for $n \leqslant \omega$ is defined as the generic directed graph on which $\perp$ is an equivalence relation with $n$ classes.

Theorem 5.4. $D\left(n * I_{\infty}\right)=\omega$ if $n=1$, and 2 for $n \geqslant 2$. 
Proof. Clearly $D\left(I_{\infty}\right)=\omega$. Now for $n>2$, pick any vertex $a$ and let $T=a^{\prime}$. Then one can verify that $(a, T)$ is a fixing type. It is interesting to verify item 4 of Definition 2.6 that one must in some cases use $n \geqslant 3$ to find a suitable vertex of $T$. For $n=2$, pick any vertex $a$ and $b \in^{\prime} a$, and let $T=a^{\prime} \cup b^{\prime}$. Then one can verify that $(\{a, b\}, T)$ is a fixing type.

Finally there is a variant, called the semigeneric graph which we write as $D\left(n \stackrel{s}{*} I_{\infty}\right)$, for which the following additional constraint is imposed: for any pairs of two vertices $A_{1}$ and two vertices $A_{2}$ taken from distinct $\perp$-classes, the number of edges from $A_{1}$ to $A_{2}$ is even. Interestingly we get the following:

Theorem 5.5. $D\left(n \stackrel{s}{*} I_{\infty}\right)=\omega$ if $n=1,2$, and 2 for $n \geqslant 3$.

Proof. Again easily $D\left(I_{\infty}\right)=\omega$, but the case $n=2$ is already interesting. For this consider any labeling and vertex $a$. Given any two vertices $x$ and $y$ in ' $a$, then the parity condition ensures that for any other vertex $b, b \in^{\prime} x$ iff $b \in^{\prime} y$. So if $x$ and $y$ were to receive the same label, then they could be interchanged to produce a non-trivial automorphism.

Now for $n \geqslant 2$, the proof carries almost identically as the above.

\subsection{Exceptional graphs}

The first exceptional homogeneous directed graph is the universal partial order $\mathbb{P}$ (viewed as a directed graph). It is the amalgamation of the class of all finite partial orders. The partial order $\mathbb{P}$ does not have free amalgamation since the amalgamation of two structures may require some additional relations to obey the transitive nature of the order relation. But we will show that it has a fixing type, and therefore has distinguishing number 2 .

Theorem 5.6. $D(\mathbb{P})=2$.

Proof. Let $G$ be the automorphism group of $\mathbb{P}=(P, \leqslant)$. For two elements $a$ and $b$ in $P$ we say that $a$ and $b$ are not related and again write $a \perp b$ if $a$ and $b$ are incomparable, that is $a \neq b$ and $\neg(a<b)$ and $\neg(b<a)$. Fix $p \in P$ and let $T$ be the set of all elements $t \in P$ with $p \perp t$. We will show that $(p, T)$ is a fixing type of $G$ acting on $P$ using the trivial partition $P=\left(P_{0}\right)$. Only items 2 and 4 need to be verified.

Item 2: $(p, T)$ is easily seen to be an extended set type. For the cover property, we have to show that if $S$ is a finite subset of $P \backslash\{p\}$ then there are infinitely many elements $t \in T$ with $\neg(t \perp s)$ for all $s \in S$. Let $\mathfrak{L}=(S \cup\{p\}, \leqslant)$ be a linear extension of the partial order induced by $\mathbb{P}$ on $S \cup\{p\}$. Now let $u$ be an element not in $P$ and $\mathfrak{X}=(S \cup\{p, u\}, \leqslant)$ the partial order so that $\mathfrak{X}$ restricted to $S \cup\{p\}$ is equal to $\mathbb{P}$ restricted to $S \cup\{p\}, u<s$ in $\mathfrak{X}$ if $p<s$ in $\mathfrak{L}$ and $u>s$ in $\mathfrak{X}$ if $p>s$ in $\mathfrak{L}$, and $u \perp p$. By the mapping extension property, there are infinitely many embeddings of $\mathfrak{X}$ into $\mathbb{P}$ which fix $\{p\} \cup S$.

Item 4: Let $a, b \in P \backslash(T \cup\{p\})$ with $a \neq b$. Both $a$ and $b$ must be related to $p$. If $a<p$ and $b>p$, or if $a>p$ and $b<p$, then clearly $\neg(a \stackrel{(T)}{\sim} b)$ as $g(p)=p$ for $g \in G_{(T)}$. Hence we may assume without loss of generality that $a<p$ and $b<p$ and $b \nless a$. Let $x$ be an 
element not in $P$ and $\mathfrak{X}=(\{p, a, b, x\}, \leqslant)$ the partial order so that the restriction of $\mathfrak{X}$ to $\{p, a, b\}$ is equal to the restriction of $\mathbb{P}$ to $\{p, a, b\}, x \perp p, x>a$, and $x \perp b$. Since there is an embedding $f$ of $\mathfrak{X}$ which fixes $\{p, a, b\}$ and maps $x$ into $T$, then $\neg(a \stackrel{(f(x))}{\sim} b)$.

This completes the proof of Theorem 5.6.

A very peculiar example of a homogeneous directed graph and the second exceptional case is the twisted universal partial order $\mathbb{P}^{*}$ (see [2], [3] where it is called $\mathcal{P}(3)$ ).

To describe $\mathbb{P}^{*}$, first partition the domain $P$ of the universal partial order $\mathbb{P}$ into three dense subsets $P_{i}$ indexed by the integers modulo 3 . Identifying the three binary relations holding in $\mathbb{P}$ with the integers modulo 3 (for example identify $<, \perp$, $>$ with $0,1,2$ respectively), let $\mathbb{P}^{\prime}$ be the variant of $\mathbb{P}$ obtained by shifting the cross types in $P_{i} \times P_{j}$ by $j-i$ modulo 3 . Finally the twisted partial order $\mathbb{P}^{*}$ is the directed graph on $P \cup\{a\}$ characterized by $\left(a^{\perp}, a^{\prime},{ }^{\prime} a\right)=\mathbb{P}^{\prime}$. It is proved in [2] that $\mathbb{P}^{*}$ is indeed an homogeneous directed graph.

Theorem 5.7. $D\left(\mathbb{P}^{*}\right)=2$.

Proof. Let $G$ be the automorphism group of $\mathbb{P}^{*}=\left(P^{*},<^{*}\right)$, and using the notation above consider the partition $A_{0}=P_{0}$, and $A_{1}=P^{*} \backslash A_{0}$. As in the proof of Theorem 5.6, choose any $p \in P_{0}$ and let $T=\left\{b \in P_{0}: b \perp p\right\}=\left\{b \in P^{*}: b \perp\{a, p\}\right\}$. We claim that $(p, T)$ is a fixing type for $G$ and partition $\left(A_{0}, A_{1}\right)$.

For item 1, consider an element $g \in G$ and finite $S \subseteq A_{0}$ such that $g(S) \subseteq A_{0}$. Now consider a partial map $h$ on $S \cup\{a\}$ fixing $a$ and such that $h\lceil S=g\lceil S$. Then $h$ is a partial isomorphism since $a$ is perpendicular to every element of $A_{0}$. Then by homogeneity $h$ extends to an automorphism $g_{0}$ of $\mathbb{P}^{*}$. However $g_{0} \in G_{0}=G_{\left\{A_{0}\right\}}$ since $a$ is fixed and $A_{0}=P_{0}=a^{\perp}$.

Similar arguments to those of Theorem 5.6 show that the remaining items are satisfied.

There is yet another construction similar to that of $\mathbb{Q}^{*}$ above yielding a homogeneous directed graph, called $S(3)$ in [2]. It is obtained this time by partitioning $\mathbb{Q}$ into three disjoint dense sets $Q_{0}, Q_{1}$, and $Q_{2}$ and reversing edges between them. Identifying the two possible orientations of an edge with the number \pm 1 , while 0 represents the absence of an edge, shift the edges between $Q_{i}$ and $Q_{j}$ by $j-i$ modulo 3. A similar argument to Theorem 5.1 shows its distinguishing number is again $\omega$.

\subsection{Free graphs}

Consider for each $n$ the generic directed graph $\mathbb{D}_{n}$ which is the amalgamation of all finite directed graphs omitting an $n$-element independent set. For $n=2$, this is simply the universal tournament $\mathbb{T}^{\infty}$, and therefore $D\left(\mathbb{D}_{2}\right)=2$. By a similar proof, this is true in general.

Theorem 5.8. For $n \geqslant 2, D\left(\mathbb{D}_{n}\right)=2$. 
Finally let $\mathcal{T}$ be a class of finite tournaments and let $\mathcal{A}(\mathcal{T})$ be the class of directed graphs containing no embeddings of members of $\mathcal{T}$. Then $\mathcal{A}(\mathcal{T})$ has free amalgamation, and the corresponding homogeneous structure has distinguishing number two by Theorem 3.1 .

\section{Conclusion}

Of course there are many other interesting homogeneous structures, for example the "double rationals". Consider the class of finite structures equipped with two independent linear orders $\leqslant$ and $\preceq$. This is an amalgamation class and the corresponding homogeneous structure is called the double rationals, written $\mathbb{Q}_{2}$. We have already discussed that the rationals themselves have distinguishing number $\omega$, the double rationals however have distinguishing number only two.

Theorem 6.1. $D\left(\mathbb{Q}_{2}\right)=2$.

Proof. Construct a $\leqslant$-strictly increasing sequence $\left(a_{n}: n \in \mathbb{N}\right)$ which is dense in the $\preceq$ ordering, that is for any $b \prec c$, there is an $a_{n}$ in between under the $\preceq$ ordering.

Indeed given $a_{n}$ and $b \prec c$, let $x$ be an element not in $\mathbb{Q}_{2}$ and $\mathfrak{X}=\left(\left\{a_{n}, b, c, x\right\}, \leqslant, \preceq\right)$ be the structure so that the restriction of $\mathfrak{X}$ to $\left\{a_{n}, b, c\right\}$ is equal to the restriction of $\mathbb{Q}_{2}$ to $\left\{a_{n}, b, c\right\}, a_{n}<x$ and $b \preceq x \preceq c$. There is an embedding $f$ of $\mathfrak{X}$ which fixes $\left\{a_{n}, b, c\right\}$ and maps $x$ into $\mathbb{Q}_{2}$.

Let $\mathcal{B}=\left(B_{0}, B_{1}\right)$ be the partition of $\mathbb{Q}_{2}$ with $B_{0}:=\left\{a_{n}: n \in \mathbb{N}\right\}$. Then any $g \in \operatorname{Aut}\left(\mathbb{Q}_{2}\right) \downarrow \mathcal{B}$ must be the identity on $B_{0}$ since it is an increasing $\leqslant$ sequence, and the identity on $B_{1}$ by the density of $B_{0}$ in the $\preceq$ ordering.

Based on the previous examples and calculations, we conjecture that the distinguishing number of any primitive countable homogeneous relational structure is either 2 or $\omega$.

\section{References}

[1] M. O.Albertson, K. L. Collins, Symmetry Breaking in Graphs. Electron. J. Combin. 3 (1996), \#R18.

[2] G. Cherlin, The classification of countable homogeneous directed graphs and countable homogeneous $n$-tournaments. Mem. Amer. Math. Soc. 131 (1998), no. 621.

[3] G. Cherlin, Homogeneous directed graphs. The imprimitive case. Logic Colloquium '85, Elsevier 1987.

[4] R. Fraïssé, Sur l'extension aux relations de quelques propriétés des Ordres. Ann. Sci. École Norm. Sup. 71 (1954), 361-388.

[5] R. Fraïssé, Theory of Relations, Revised Edition, in : Studies in Logic and the Foundations of Mathematics, 145, North Holland 2000. 
[6] A. Gardiner, Homogeneous graphs. J. Combinatorial Theory Ser. B 20 (1976), no. 1, 94-102.

[7] C. W. Henson, Countable homogeneous relational structures and $\aleph_{0^{-} \text {-categorical }}$ theories. J. Symbolic Logic 37 (1972), 494-500.

[8] W. Imrich, S. Klavžar, Distinguishing Cartesian powers of graphs. J. Graph Theory 53 (2006), no. 3, 250-260.

[9] W. Imrich, S. Klavžar, V. Trofimov, Distinguishing Infinite Graphs. Electron. J. Combin. 14 (2007), \#R36.

[10] A. Lachlan, R. Woodrow, Countable Homogeneous Undirected Graphs. Trans. Amer. Math. Soc. 262 (1980), 51-94.

[11] N. Sauer, Partitions of countable homogeneous systems. Appendix to [5], Amsterdam 2000.

[12] J. H. Schmerl, Countable homogeneous partially ordered sets. Algebra Universalis 9 (1979), 317-321. 